\title{
Erratum: On the structure of infrared singularities of gauge-theory amplitudes
}

\section{Thomas Becher ${ }^{a}$ and Matthias Neubert ${ }^{b}$}

${ }^{a}$ Fermi National Accelerator Laboratory, P.O. Box 500, Batavia, IL 60510, U.S.A.

${ }^{b}$ Institut für Physik (THEP), Johannes Gutenberg-Universität, D-55099 Mainz, Germany

E-mail: becher@fnal.gov, neubertm@uni-mainz.de

ERratum to: JHEP06(2009)081

The expression for the quantity $\boldsymbol{H}_{\mathrm{R} . \mathrm{S} .}^{(2)}(\epsilon)$ in equation (2.17) of the paper is incomplete. The result for $\boldsymbol{H}_{\mathrm{R} . \mathrm{S} .}^{(2)}(\epsilon)$ must include the additional term

$$
\Delta \boldsymbol{H}_{\mathrm{R} . \mathrm{S} .}^{(2)}(\epsilon)=-\frac{i f^{a b c}}{128 \epsilon} \gamma_{0}^{\text {cusp }} \sum_{(i, j, k)} \boldsymbol{T}_{i}^{a} \boldsymbol{T}_{j}^{b} \boldsymbol{T}_{k}^{c}\left(\frac{\gamma_{0}^{i}}{C_{i}}-\frac{\gamma_{0}^{j}}{C_{j}}\right) \ln \frac{-s_{i j}}{-s_{j k}} \ln \frac{-s_{k i}}{-s_{i j}} .
$$

This extra contribution was discussed in appendix D of [1], where it was shown that it only contributes for amplitudes with more than four particles. The form (2.17) of the extra term was given in the recent paper [2].

\section{References}

[1] S.M. Aybat, L.J. Dixon and G.F. Sterman, The two-loop soft anomalous dimension matrix and resummation at next-to-next-to leading pole, Phys. Rev. D 74 (2006) 074004 [hep-ph/0607309] [INSPIRE].

[2] T. Becher, G. Bell, C. Lorentzen and S. Marti, Transverse-momentum spectra of electroweak bosons near threshold at NNLO, arXiv:1309.3245 [INSPIRE]. 